\title{
A Bound for the Joint Spectral Radius of Operators in a Hilbert Space
}

\author{
Michael Gil ${ }^{1 *}$ \\ ${ }^{1}$ Department of Mathematics, Ben Gurion University of the Negev, Beer-Sheva 84105, Israel \\ ${ }^{*}$ Corresponding author
}

\section{Article Info}

Keywords: Joint spectral radius, Hilbert space, Discrete time switched systems

2010 AMS: 47A10, 93C30, 93C55, 93D05, $15 A 18$

Received: 25 March 2019

Accepted: 13 May 2019

Available online: 28 June 2019

\begin{abstract}
We suggest a bound for the joint spectral radius of a finite set of operators in a Hilbert space. In appropriate situations that bound enables us to avoid complicated calculations and gives a new explicit stability test for the discrete time switched systems. The illustrative example is given. Our results are new even in the finite dimensional case.
\end{abstract}

\section{Introduction and statement of the main result}

Let $\mathscr{H}$ be a complex separable Hilbert space with a scalar product $(.,$.$) , the norm \|\|=.\sqrt{(., .)}$ and unit operator $I$. By $\mathscr{B}(\mathscr{H})$ we denote the set of all bounded linear operators in $\mathscr{H}$. For an $A \in \mathscr{B}(\mathscr{H}), \sigma(A)$ is the spectrum, $r_{s}(A)$ is the spectral radius; $A^{*}$ is adjoint to $A$, and $\|A\|=\sup _{h \in \mathscr{H}, h \neq 0}\|A h\| /\|h\|$.

Let $\mathscr{M}=\left\{A_{1}, \ldots, A_{v}\right\}$ be a finite set of operators $A_{j} \in \mathscr{B}(\mathscr{H})(j=1, \ldots, v)$. Our main object is the joint spectral radius $\rho(\mathscr{M})$ of $\mathscr{M}$ defined by

$$
\rho(\mathscr{M}):=\lim _{k \rightarrow \infty} \sup \left\{\left\|A_{i_{k}} \cdots A_{i_{1}}\right\|^{1 / k}: A_{i} \in \mathscr{M}\right\},
$$

cf. [1,2]. The joint spectral radius arises naturally in a range of topics including the theory of difference equations [3], control and stability of discrete time switched systems [4, 5, 6, 7, 8, 9, 10, 11], wavelets [12], ergodic theory [13], etc.

The literature on the theory of the joint spectral radius is rather rich, cf. [14], [15], [16], [17, 18] and references therein. Mainly, the finite dimensional operators were considered and the numerical methods were developed.

In the present paper, under some restrictions, we suggest a bound for $\rho(\mathscr{M})$. In appropriate situations that bound enables us to avoid complicated calculations and gives an explicit stability test for the discrete time switched systems. The example characterizing the sharpness of our results is given. To the best of our knowledge, our results are new even in the finite dimensional case.

Let $A \in \mathscr{B}(\mathscr{H})$ with $r_{s}(A)<1$. Then the discrete Lyapunov equation

$$
X-A^{*} X A=I
$$

has a positive definite self-adjoint solution $X(A)$ [19]. It can be represented by

$$
X(A)=\sum_{j=0}^{\infty}\left(A^{*}\right)^{j} A^{j}
$$

and

$$
X(A)=\frac{1}{2 \pi} \int_{0}^{2 \pi}\left(I e^{-i \omega}-A^{*}\right)^{-1}\left(I e^{i \omega}-A\right)^{-1} d \omega,
$$

cf. [20, Section 7.1]. We will say that $\mathscr{M}$ is Schur-Kohn stable, if $\rho(\mathscr{M})<1$. Now we are in a position to formulate our main result. 
Theorem 1.1. Let there be an $A \in \mathscr{B}(\mathscr{H})$ with $r_{s}(A)<1$, such that

$$
\|X(A)\|\left(2\left\|A-A_{k}\right\|\|A\|+\left\|A-A_{k}\right\|^{2}\right)<1\left(A_{k} \in \mathscr{M} ; k=1, \ldots, v\right) .
$$

Then $\mathscr{M}$ is Schur-Kohn stable. Moreover,

$$
\rho(\mathscr{M}) \leq \sqrt{1-\frac{1}{\|X(A)\|}\left(1-\max _{j=1, \ldots, v}\|X(A)\|\left(2\left\|A-A_{j}\right\|\|A\|+\left\|A-A_{j}\right\|^{2}\right)\right.} .
$$

The proof of this theorem is presented in the next section. In Theorem 1.1, one can take $A=A_{m}$ for an $A_{m} \in \mathscr{M}$. Below we consider some concrete classes of operators. Note that from (1.2) and (1.3) it follows that

$$
\|X(A)\| \leq \sum_{j=1}^{\infty}\left\|A^{j}\right\|^{2}
$$

and

$$
\|X(A)\| \leq \frac{1}{2 \pi} \int_{0}^{2 \pi}\left\|\left(I e^{i \omega}-A\right)^{-1}\right\|^{2} d \omega
$$

If $A$ is normal: $A A^{*}=A^{*} A$, then $\|A\|=r_{s}(A)$ and (1.5) implies

$$
\|X(A)\| \leq \sum_{j=0}^{\infty} r_{s}^{2 j}(A)=\frac{1}{1-r_{s}^{2}(A)} .
$$

\section{Proof of Theorem 1.1}

In this section for the simplicity we put $X(A)=X$.

Lemma 2.1. Let $A, \tilde{A} \in \mathscr{B}(\mathscr{H}), r_{S}(A)<1$ and $X$ be a solution of (1.1). If

$$
\|X\|\left(2\|A-\tilde{A}\|\|A\|+\|A-\tilde{A}\|^{2}\right)<1,
$$

then

$$
(X \tilde{A} x, \tilde{A} x) \leq\left(1-\frac{c_{0}}{\|X\|}\right)(X x, x)(x \in \mathscr{H})
$$

where

$$
c_{0}:=1-\|X\|\left(2\|A-\tilde{A}\|\|A\|+\|A-\tilde{A}\|^{2}\right) .
$$

Proof. Put $Y=\tilde{A}-A$. Then

$$
X-\tilde{A}^{*} X \tilde{A}=X-(Y+A)^{*} X(Y+A)=X-A^{*} X A-Y^{*} X A-A^{*} X Y-Y^{*} X Y=I-Y^{*} X A-A^{*} X Y-Y^{*} X Y .
$$

By (2.1)

$$
\left\|I-Y^{*} X A-A^{*} X Y-Y X Y\right\| \geq 1-\left\|Y^{*} X A-A^{*} X Y-Y^{*} X Y\right\|, \geq 1-\|X\|\left(2\|A-\tilde{A}\|+\|A-\tilde{A}\|^{2}\right)=c_{0} .
$$

Thus,

$$
X-\tilde{A}^{*} X \tilde{A} \geq c_{0} I
$$

Hence,

$$
(X x, x)-(X \tilde{A} x, \tilde{A} x) \geq c_{0}(x, x) \geq c_{0}\left(\frac{X}{\|X\|} x, x\right)=\frac{c_{0}}{\|X\|}(X x, x),
$$

as claimed.

Proof of Theorem 1.1: Define the norms

$$
|x|_{X}=\sqrt{(X x, x)}(x \in \mathscr{H}) \text { and }|A|_{X}=\sup _{x \in \mathscr{H}} \frac{|A x|_{X}}{|x|_{X}} .
$$

Due to Lemma 2.1 and (1.4) we have

$$
\left|A_{j}\right|_{X}^{2} \leq 1-\frac{c_{j}}{\|X\|}
$$

where

$$
c_{j}:=1-\|X\|\left(2\left\|A-A_{j}\right\|\|A\|+\left\|A-A_{j}\right\|^{2}\right) .
$$


Put

$$
a_{0}:=\max _{j} \sqrt{1-\frac{c_{j}}{\|X\|}}=\sqrt{1-\frac{1}{\|X\|}\left(1-\max _{j}\|X\|\left(2\left\|A-A_{j}\right\|\|A\|+\left\|A-A_{j}\right\|^{2}\right)\right.}
$$

Then by (2.1)

$$
\max _{j}\left|A_{j}\right|_{X} \leq a_{0}
$$

Since $X$ is positive definite, it is boundedly invertible. For any $T \in \mathscr{B}(\mathscr{H})$ one has

$$
\frac{\|T x\|^{2}}{\|x\|^{2}}=\frac{\left(X^{-1} X T x, T x\right)}{\left(X^{-1} X x, x\right)} \leq \frac{\left(X^{-1} X T x, T x\right)}{\left(\frac{1}{\|X\|} X x, x\right)} \leq\|X\|\left\|X^{-1}\right\| \frac{(X T x, T x)}{(X x, x)}(x \in \mathscr{H}) .
$$

So

$$
\|T\|^{2} \leq\|X\|\left\|X^{-1}\right\||T|_{X}^{2}
$$

Hence, according to (2.2),

$$
\left\|A_{i_{k}} \cdots A_{i_{1}}\right\| \leq\left(\|X\|\left\|X^{-1}\right\|\right)^{1 / 2}\left|A_{i_{k}} \cdots A_{i_{1}}\right| X \leq\left(\|X\|\left\|X^{-1}\right\|\right)^{1 / 2} a_{0}^{k}
$$

and therefore,

$$
\rho(\mathscr{M}) \leq \varlimsup_{k \rightarrow \infty}\left(\|X\|\left\|X^{-1}\right\|\right)^{1 / 2 k} a_{0}=a_{0},
$$

as claimed.

\section{Concrete classes of operators}

In this section we suggest estimates for $X(A)$ under various assumptions. From (1.6) it follows

$$
\|X(A)\| \leq \sup _{|z|=1}\left\|(I z-A)^{-1}\right\|^{2}
$$

Let there be monotonically increasing non-negative continuous function $F(x)(x \geq 0)$, such that $F(0)=0, F(\infty)=\infty$ and

$$
\left\|(\lambda I-A)^{-1}\right\| \leq F(1 / \operatorname{dist}(A, \lambda))(\lambda \notin \sigma(A)),
$$

where $\operatorname{dist}(A, \lambda)=\inf _{s \in \sigma(A)}|s-\lambda|$. If $r_{s}(A)<1$ and $|z|=1$, then obviously, $\operatorname{dist}(A, z) \geq 1-r_{s}(A)$ and therefore, $\left\|(I z-A)^{-1}\right\| \leq F(1 /(1-$ $\left.\left.r_{s}(A)\right)\right)$. Now (3.1) implies

$$
\|X(A)\| \leq F^{2}\left(\frac{1}{1-r_{s}(A)}\right)
$$

\subsection{Operators in finite dimensional spaces}

Let $\mathbb{C}^{n}(n<\infty)$ be the complex $n$-dimensional Euclidean space with a scalar product $(.,$.$) , the Euclidean norm \|\|=.\sqrt{(., .)}$ and unit matrix $I, \mathbb{C}^{n \times n}$ is the set of all $n \times n$ matrices. $\lambda_{k}(A), k=1, \ldots, n$, are the eigenvalues of $A \in \mathbb{C}^{n \times n}$, counted with their multiplicities; $N_{2}(A)=\left(\text { trace } A A^{*}\right)^{1 / 2}$ is the Hilbert-Schmidt (Frobenius) norm of $A$. The quantity (the departure from normality of $A$ )

$$
g(A)=\left[N_{2}^{2}(A)-\sum_{k=1}^{n}\left|\lambda_{k}(A)\right|^{2}\right]^{1 / 2}
$$

plays an essential role hereafter. The following relations are checked in [21, Section 3.1]:

$$
g^{2}(A) \leq N_{2}^{2}(A)-\left|\operatorname{trace} A^{2}\right| \text { and } g^{2}(A) \leq \frac{N_{2}\left(A-A^{*}\right)}{2}=2 N_{2}^{2}\left(A_{I}\right),
$$

where $A_{I}=\left(A-A^{*}\right) / 2 i$. If $A$ is a normal matrix, then $g(A)=0$.

Due to Example 3.3 from [21],

$$
\left\|A^{m}\right\| \leq \sum_{k=0}^{n-1} \frac{m ! r_{s}^{m-k}(A) g^{k}(A)}{(m-k) !(k !)^{3 / 2}} \quad(m=1,2, \ldots) .
$$

Now (1.5) implies

$$
\|X(A)\| \leq \xi_{n}(A):=\sum_{j=1}^{\infty}\left(\sum_{k=0}^{n-1} \frac{m ! r_{s}^{m-k}(A) g^{k}(A)}{(m-k) !(k !)^{3 / 2}}\right)^{2} \quad\left(A \in \mathbb{C}^{n \times n}\right) .
$$

Note that if $A$ is normal, then $g(A)=0$ and (3.3) gives us the sharp inequality (1.7).

Theorem 1.1 and (3.3) yield the following corollary. 
Corollary 3.1. Let $\mathscr{M}$ be a finite set of $n \times n$-matrices. Let there be an $n \times n$-matrix $A$ with $r_{s}(A)<1$, such that

$$
\xi_{n}(A) \max _{B \in \mathscr{M}}\left(2\|A-B\|\|A\|+\|A-B\|^{2}\right)<1 .
$$

Then $\mathscr{M}$ is Schur-Kohn stable. Moreover,

$$
\rho(\mathscr{M}) \leq \sqrt{1-\frac{1}{\xi_{n}(A)}\left(1-\xi_{n}(A) \max _{B \in \mathscr{M}}\left(2\|A-B\|\|A\|+\|A-B\|^{2}\right)\right.} .
$$

Let us point the more compact but less sharper estimate for $X(A)$. To this end put

$$
\eta_{n}(A):=\sum_{k=0}^{n-1} \frac{g^{k}(A)}{\sqrt{k !}\left(1-r_{s}(A)\right)^{k+1}} .
$$

By Theorem 3.2 from [21]

$$
\left\|(A-\lambda I)^{-1}\right\| \leq \sum_{k=0}^{n-1} \frac{g^{k}(A)}{(\operatorname{dist}(A, \lambda))^{k+1} \sqrt{k !}} \quad\left(A \in \mathbb{C}^{n \times n}, \lambda \notin \sigma(A)\right) .
$$

Making use of (3.2) we can assert that

$$
\|X(A)\| \leq \eta_{n}^{2}(A)\left(A \in \mathbb{C}^{n \times n}\right) .
$$

So in Corollary 3.1 one can replace $\xi_{n}(A)$ by $\eta_{n}^{2}(A)$.

\subsection{Hilbert-Schmidt operators}

Denote by $S N_{2}$ the ideal of Hilbert-Schmidt operators in $\mathscr{H}$ with the finite norm $N_{2}(A)=\left(\text { trace } A A^{*}\right)^{1 / 2}$. In the infinite dimensional case we put

$$
g(A)=\left[N_{2}^{2}(A)-\sum_{k=1}^{\infty}\left|\lambda_{k}(A)\right|^{2}\right]^{1 / 2},
$$

where $\lambda_{k}(A), k=1,2, \ldots$, are the eigenvalues of $A \in S N_{2}$, counted with their multiplicities and enumerated in the non-increasing order of their absolute values.

Since

$$
\sum_{k=1}^{\infty}\left|\lambda_{k}(A)\right|^{2} \geq\left|\sum_{k=1}^{\infty} \lambda_{k}^{2}(A)\right|=\left|\operatorname{trace} A^{2}\right|
$$

one can write

$$
g^{2}(A) \leq N_{2}^{2}(A)-\left|\operatorname{trace} A^{2}\right| .
$$

If $A$ is a normal Hilbert-Schmidt operator, then $g(A)=0$, since

$$
N_{2}^{2}(A)=\sum_{k=1}^{\infty}\left|\lambda_{k}(A)\right|^{2}
$$

in this case. Moreover,

$$
g^{2}(A) \leq \frac{N_{2}^{2}\left(A-A^{*}\right)}{2}=2 N_{2}^{2}\left(A_{I}\right)
$$

cf. [21, Section 7.1]. Due to Corollary 7.4 from [21] for any $A \in S N_{2}$ we have

$$
\left\|A^{m}\right\| \leq \sum_{k=0}^{m} \frac{m ! r_{s}^{m-k}(A) g^{k}(A)}{(m-k) !(k !)^{3 / 2}} \quad(m=1,2, \ldots) .
$$

Now (1.5) implies

$$
\|X(A)\| \leq \xi_{\infty}(A):=\sum_{j=1}^{\infty}\left(\sum_{k=0}^{m} \frac{m ! r_{s}^{m-k}(A) g^{k}(A)}{(m-k) !(k !)^{3 / 2}}\right)^{2} \quad\left(A \in S N_{2}\right) .
$$

If $A$ is normal, then (3.4) gives us inequality (1.7).

Furthermore, by Theorem 7.1 from [21] for any $A \in S N_{2}$ we have

$$
\left\|R_{\lambda}(A)\right\| \leq \sum_{k=0}^{\infty} \frac{g^{k}(A)}{(\operatorname{dist}(A, \lambda))^{k+1} \sqrt{k !}}(\lambda \notin \sigma(A)) .
$$

Inequality (3.2) gives us the more compact but less sharper estimate

$$
\|X(A)\| \leq \eta_{\infty}^{2}(A)\left(A \in S N_{2}\right),
$$


where

$$
\eta_{\infty}(A):=\sum_{j=0}^{\infty} \frac{g^{j}(A)}{\sqrt{j !}\left(1-r_{s}(A)\right)^{j+1}} .
$$

Now we can directly apply Theorem 1.1 .

By the Schwarz inequality

$$
\begin{aligned}
& \left(\sum_{j=0}^{\infty} \frac{(c g)^{j}(A)}{c^{j} \sqrt{j !}\left(1-r_{s}(A)\right)^{j}}\right)^{2} \leq \sum_{k=0}^{\infty} c^{2 k} \sum_{j=0}^{\infty} \frac{g^{2 j}(A)}{c^{2 j} j !\left(1-r_{s}(A)\right)^{2 j}}= \\
& \frac{1}{1-c^{2}} \exp \left[\frac{g^{2}(A)}{c^{2}\left(1-r_{s}(A)\right)^{2}}\right] \quad(c \in(0,1)) .
\end{aligned}
$$

Thus,

$$
\|X(A)\| \leq \frac{1}{\left(1-c^{2}\right)\left(1-r_{s}(A)\right)^{2}} \exp \left[\frac{g^{2}(A)}{c^{2}\left(1-r_{s}(A)\right)^{2}}\right] \quad\left(A \in S N_{2}, c \in(0,1)\right) .
$$

In particular, taking $c^{2}=1 / 2$, we get

$$
\|X(A)\| \leq \hat{\eta}(A):=\frac{2}{\left(1-r_{s}(A)\right)^{2}} \exp \left[\frac{2 g^{2}(A)}{\left(1-r_{S}(A)\right)^{2}}\right] .
$$

Now Theorem 1.1 implies the following corollary.

Corollary 3.2. Let $\mathscr{M}$ be a finite set of bounded operators from $\mathscr{H}$ Let there be an $A \in S N_{2}$ with $r_{s}(A)<1$, such that

$$
\hat{\eta}(A) \max _{B \in \mathscr{M}}\left(2\|A-B\|\|A\|+\|A-B\|^{2}\right)<1 .
$$

Then $\mathscr{M}$ is Schur-Kohn stable. Moreover,

$$
\rho(\mathscr{M}) \leq \sqrt{1-\frac{1}{\hat{\eta}(A)}\left(1-\hat{\eta}(A) \max _{B \in \mathscr{M}}\left(2\|A-B\|\|A\|+\|A-B\|^{2}\right)\right.} .
$$

Similarly, making use of Theorems 7.2, 7.3 from [21] one can apply Theorem 1.1 to Shatten-von Neumann operators.

\subsection{Non-compact non-normal operators}

In this subsection we suggest a norm estimate for the solution of (1.1) under the condition

$$
A_{I}=\left(A-A^{*}\right) /(2 i) \in S N_{2} \text {. }
$$

To this end introduce the quantity

$$
g_{I}(A):=\sqrt{2}\left[N_{2}^{2}\left(A_{I}\right)-\sum_{k=1}^{\infty}\left(\mathfrak{I} \lambda_{k}(A)\right)^{2}\right]^{1 / 2} .
$$

Obviously, $g_{I}(A) \leq \sqrt{2} N_{2}\left(A_{I}\right)$. Due to Example 10.2 from [21],

$$
\left\|A^{m}\right\| \leq \sum_{k=0}^{m} \frac{m ! r_{s}^{m-k}(A) g_{I}^{k}(A)}{(m-k) !(k !)^{3 / 2}} \quad(m=1,2, \ldots) .
$$

Now (1.5) implies

$$
\|X(A)\| \leq \xi_{I}(A):=\sum_{j=0}^{\infty}\left(\sum_{k=0}^{m} \frac{m ! r_{s}^{m-k}(A) g^{k}(A)}{(m-k) !(k !)^{3 / 2}}\right)^{2} \quad\left(A_{I} \in S N_{2}\right) .
$$

If $A$ is normal from this inequality we get (1.7).

Furthermore, by Theorem 9.1 from [21] under condition (4.1) we have,

$$
\left\|R_{\lambda}(A)\right\| \leq \sum_{k=0}^{\infty} \frac{g_{I}^{k}(A)}{(\operatorname{dist}(A, \lambda))^{k+1} \sqrt{k !}}
$$

and

$$
\left\|R_{\lambda}(A)\right\| \leq \frac{\sqrt{e}}{\operatorname{dist}(A, \lambda)} \exp \left[\frac{g_{I}^{2}(A)}{2(\operatorname{dist}(A, \lambda))^{2}}\right] \quad(\lambda \notin \sigma(A)) .
$$

Inequality (3.2) implies

$$
\|X(A)\| \leq \eta_{I}^{2}(A) \text { and }\|X(A)\| \leq \hat{\eta}_{I}^{2}(A)\left(A_{I} \in S N_{2}\right),
$$

where

$$
\eta_{I}:=\sum_{j=0}^{\infty} \frac{g_{I}^{j}(A)}{\sqrt{j !}\left(1-r_{s}(A)\right)^{j+1}}
$$


and

$$
\hat{\eta}_{I}:=\frac{\sqrt{e}}{1-r_{s}(A)} \exp \left[\frac{g_{I}^{2}(A)}{2\left(1-r_{s}(A)\right)^{2}}\right] .
$$

Now we can directly apply Theorem 1.1 .

Some other classes of operators can be considered, in particular, via norm estimates for operator functions from [21].

\section{Example}

The following example characterizes the sharpness of Theorem 1.1 .

Let $\mathscr{H}=\mathbb{C}^{n}, \mathscr{M}=\left\{A_{1}, A_{2}\right\}$ with real positive matrices matrices $A_{1}=\operatorname{diag}\left(a_{k}\right)_{k=1}^{n}, A_{2}=\operatorname{diag}\left(b_{k}\right)_{k=1}^{n} ;\left(a_{k}, b_{k} \geq 0\right)$ and $r_{s}\left(A_{1}\right)<1$. So $a_{m}=\max _{k} a_{k}<1$. Take $A=A_{1}$. Since $A_{1}$ is Hermitian, according to (1.7) condition (1.4) takes the form

$$
\frac{1}{1-r_{s}^{2}\left(A_{1}\right)}\left(2 r_{s}\left(A_{1}\right)\left\|A_{1}-A_{2}\right\|+\left\|A_{1}-A_{2}\right\|^{2}\right)<1
$$

Besides, $\left\|A_{1}-A_{2}\right\|=\max _{k}\left|a_{k}-b_{k}\right|$.

Assume that $r_{s}\left(A_{2}\right) \geq 1$. Namely, $b_{m} \geq 1$. So $\mathscr{M}$ is Schur-Kohn unstable. Then $\left|a_{m}-b_{m}\right|=b_{m}-a_{m} \geq 1-a_{m}$ and

$$
\frac{1}{1-a_{m}^{2}}\left(2 a_{m}\left|a_{m}-b_{m}\right|+\left|a_{m}-b_{m}\right|^{2}\right) \geq \frac{1}{1-a_{m}^{2}}\left(2 a_{m}\left(1-a_{m}\right)+\left(1-a_{m}\right)^{2}\right) \geq \frac{1}{1+a_{m}}\left(2 a_{m}+1-a_{m}\right) \geq 1 .
$$

Therefore, condition (4.1) is not fulfilled. So condition (1.4) is necessary under consideration.

\section{Acknowledgement}

I am very grateful to the referees of this article for their helpful remarks.

\section{References}

[1] G. C. Rota and G. Strang. A note on the joint spectral radius, Proc. Netherlands Acad., 22 (1960) 379-381

[2] R. Jungers, The Joint Spectral Radius: Theory and Applications, Springer, Berlin, 2009.

[3] V. Kozyakin, On the computational aspects of the theory of joint spectral radius, Dokl. Akad. Nauk, 427 (2009), 160-164, in Russian, translation in Doklady Mathematics, 80 (2009), 487-491.

[4] X. Dai, Y. Huang and M. Xiao, Almost sure stability of discrete-time switched linear systems: A topological point of view, SIAM J. Control Optim., 47 (2008), 2137-156.

[5] J.-W. Lee and G. E. Dullerud, Uniform stabilization of discrete-time switched and Markovian jump linear systems, Automatica, 42 (2), (2006) 205-218.

[6] D. Liberzon, Switching in Systems and Control. Birkháuser, Boston, 2003.

[7] H. Lin and P. J. Antsaklis, Stability and stabilizability of switched linear systems: a survey of recent results, IEEE Transac. on Automatic Control, 54 (2), (2009) 308-322.

[8] R. Shorten, F. Wirth, O. Mason, K.Wul and C. King, Stability criteria for switched and hybrid systems, SIAM Rev., 49 (2007), $545-592$.

[9] W. Xiang and J. Xiao, Convex sufficient conditions on asymptotic stability and l2 gain performance for uncertain discrete-time switched linear systems. IET Control Theory Appl. 8 (3), (2014), 211-218.

[10] G. Zhai and X. Xu, A unified approach to stability analysis of switched linear descriptor systems under arbitrary switching, Int. J. Appl. Math. Comput. Sci., 20 (2), (2010), 249-259.

[11] L. Zhang, Y. Zhu, P. Shi, Q. Lu, Time-Dependent Switched Discrete-Time Linear Systems: Control and Filtering Springer International Publishing, Switzerland, 2016

[12] C. Heil and G. Strang, Continuity of the joint spectral radius: Applications to wavelets, in "Linear Algebra for Signal Processing," IMA Vol. Math. Appl. 69, Springer-Verlag, New York, (1995), 51-61.

[13] I. D. Morris, A rapidly-converging lower bound for the joint spectral radius via multiplicative ergodic theory, Adv. Math., 225 (2010), $3425-3445$.

[14] V. Kozyakin, On accuracy of approximation of the spectral radius by the Gelfand formula, Linear Algebra Appl., 431 (2009), $2134-2141$.

[15] V. Kozyakin, A relaxation scheme for computation of the joint spectral radius of matrix sets, J. Difference Equ. Appl., 17 (2), (2011) $185-201$.

[16] V. D. Blondel and Y. Nesterov, Polynomial-time computation of the joint spectral radius for some sets of nonnegative matrices, SIAM J. Matrix Anal. Appl., 31 (2009), 865-876.

[17] P.A. Parrilo and A. Jadbabaie, Approximation of the joint spectral radius using sum of squares, Linear Algebra Appl., 428 (2008), 2385-2402

[18] J. N. Tsitsiklis and V. D. Blondel, The Lyapunov exponent and joint spectral radius of pairs of matrices are hard when not impossible-to compute and to approximate, Math. Control, Signals and Systems, 10 (1997), 31-40.

[19] T. Eisner, Stability of Operators and Operator Semigroups, Operator Theory: Advances and Applications Vol. 209, Birkhăuser Verlag, Basel, 2010.

[20] M.I. Gil'. Difference Equations in Normed Spaces. Stability and Oscillations, North-Holland, Mathematics Studies 206, Elsevier, Amsterdam, 2007.

[21] M.I. Gil', Operator Functions and Operator Equations, World Scientific, New Jersey, 2018. 